\title{
Nichtnatürliche Todesfälle und Verletzungen im Rahmen von Mortalität und Morbidität
}

\author{
Von W. Casper
}

\section{Zusammenfassung}

Der Anteil von Verletzungen am Mortalitäts- und Morbiditätsgeschehen wird generell unterschätzt. Gemessen an den in Zahl und Häufigkeit bedeutenden Krankheiten des Kreislaufsystems und den bösartigen Neubildungen scheinen sie von untergeordneter Bedeutung. Verletzungen verursachen zwar nur rd. $5 \%$ aller Sterbefälle, berücksichtigt man jedoch die hohen Verluste an potentiellen Lebensjahren und das niedrige mittlere Sterbealter, gehören Verletzungen zu den drei wichtigsten Todesursachen. Im Kindes-, Jugend- und jüngeren Erwachsenenalter bestimmen Verletzungen das gesamte Sterbegeschehen. Die Ursachen tödlicher Verletzungen sind zu $60 \%$ Unfälle, zu $33 \%$ Suizide und zu $2 \%$ Gewalttätigkeiten durch andere Personen. Verletzungen verursachen rd. $10 \%$ der Krankenhausbehandlungsfälle. Gemeinsam mit den bösartigen Neubildungen liegen sie hinter den HerzKreislaufkrankheiten an zweiter Stelle der stationären Behandlungshäufigkeiten. Täglich sind rd. 5100 Krankenhausbetten mit Verletzten belegt. In der Öffentlichkeit werden Verletzungen meist nur aus Polizeiberichten als Verkehrsunfälle und Gewalttätigkeiten durch eine andere Person wahrgenommen, selten unter dem Aspekt der Prävention. Dabei ist eine ursachenspezifische Prävention insbesondere bei den Unfällen möglich, wie viele Beispiele aus anderen Ländern zeigen. Die häufiger werdenden Gewalttätigkeiten gegenüber anderen Personen sind Ausdruck wachsender sozialer Spannungen. Ihre Prävention ist eine gesellschaftliche Aufgabe von hohem Rang.

\section{Summary \\ Unnatural Deaths and Injuries as Morbidity and Mortality Parameters}

The share of injuries among mortality and morbidity figures is generally underestimated. In relation to the diseases of the cardio-vascular system and of malignant neoplasms, which are important by their rate and frequency, they seem to be of subordinate significance. Though injuries cause only about $5 \%$ of deaths, they belong to the three outstanding causes of death considering the high number of potential years of life lost and the low mean age at death. Injuries are the most ominent cause of death in childhood age, adolescence and early adult years. Causes of lethal injuries are accidents $(60 \%)$, suicide $(33 \%)$ and violence by other persons $(2 \%)$. Injuries are the cause of about $10 \%$ of cases requiring hospital treatment. Together with cases of malignant neoplasms, they figure in second position after cardiovascular diseases on the frequency scale of hospital treatment. Every day about 5100 hospital beds are occupied by injured persons. Public attention mostly recognizes injuries in police reports as a result of traffic accidents and violence caused by another person, but rarely considers them under the aspect of prevention. However, particularly with regard to accidents, cause-related prevention at the source is possible as has been demonstrated by many examples from other countries. Social tensions find their expression by increasing violence towards other persons. The prevention of this phenomenon should be a social challenge of high priority.

\section{Einleitung}

Verletzungen sind die Folgen von gewaltsamen äußeren Einwirkungen. Sie können verschiedene Ursachen haben, z. B. einen Unfall, eine Gewalteinwirkung durch eine andere Person oder einen Suizid bzw. Suizidversuch. Die medizinische Dokumentation der Verletzungen erfolgt nach den Diagnosen der Klasse XVII "Verletzungen und Vergiftungen « der Internationalen Klassifikation der Krankheiten, Verletzungen und Todesursachen (ICD-9, Dg.-Nr. 800-999). Für tödlich endende Verletzungen (nichtnatürliche Todesfälle) werden darüber hinaus die Ursachen nach der zusätzlichen Klassifikation der äußeren Ursachen von Verletzungen und Vergiftungen (E-Klassifikation, Nr. E800-E999) und der Ereignisort auf dem Leichenschauschein dokumentiert.
Dadurch sind zumindest für die Gestorbenen Aussagen über das Ausmaß und die Häufigkeit der jeweiligen Ursachen (Unfall, Suizid, Mord etc.) möglich. EKlassifikation und Ereignisort geben insbesondere bei den Unfällen Hinweise

auf spezifische Risiken und Ansatzpunkte für Präventionsmaßnahmen.

Verletzungen spielen wegen ihrer Häufigkeit im Morbiditäts- und Mortalitätsgeschehen vor allem im Kindes- und jüngeren Erwachsenenalter eine große

Tabelle 1: Verletzte und nichtnatürliche Sterbefälle nach Ursachen

\begin{tabular}{|c|c|c|}
\hline & $\begin{array}{r}\text { Verletzte } \\
\text { (z. T. geschätzt) }\end{array}$ & $\begin{array}{r}\text { Gestorbene } \\
\text { (1995) }\end{array}$ \\
\hline Haus- und Freizeitunfälle & 5,0 Mio. & 13500 \\
\hline Arbeits- und Wegeunfälle & 2,0 Mio. & 800 \\
\hline Schulunfälle* & 1,5 Mio. & \\
\hline Verkehrsunfälle & 400000 & 9500 \\
\hline Suizid und Selbstbeschädigung & 70000 & 12900 \\
\hline Gewalteinwirkungen (Mord) & 300000 & 1200 \\
\hline (unbekannt) & & 1700 \\
\hline
\end{tabular}

* Unfälle von Schülern, Studenten und Kindern in Kindergärten 
$\%$

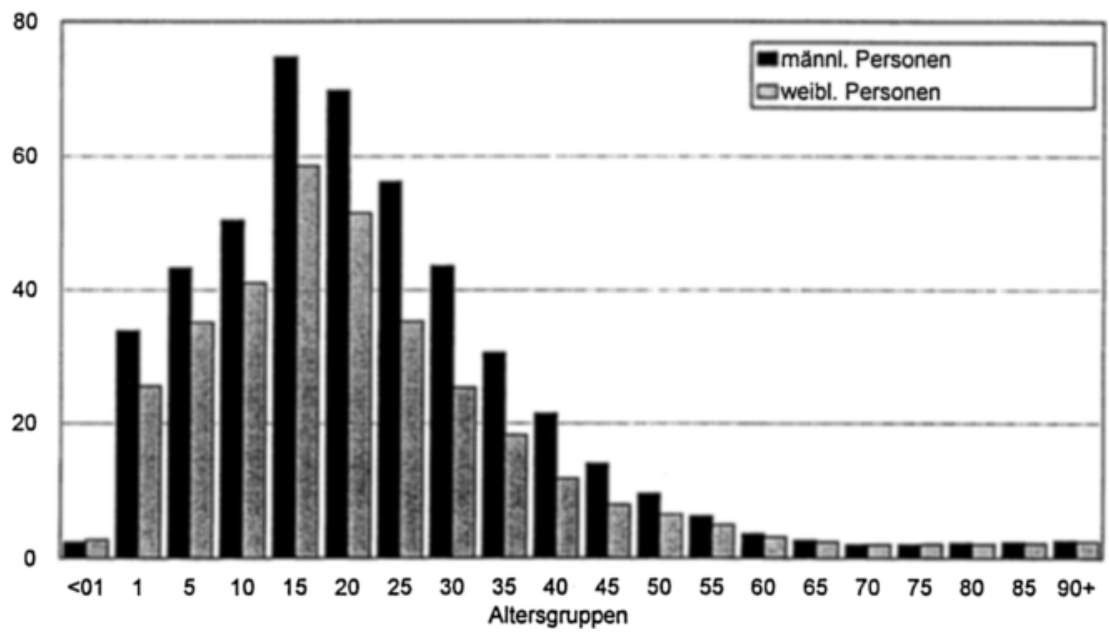

Abbildung 1: Anteile der nichtnatürlichen Sterbefälle an den Sterbefällen der Altersgruppen - Deutschland 1995.

Rolle. Von der Öffentlichkeit werden sie jedoch nicht entsprechend ihrer Bedeutung wahrgenommen. Ins öffentliche Bewußtsein dringen meist nur Polizeiberichte über Verkehrsunfälle und Gewalttätigkeiten.

In der Bundesrepublik Deutschland ist schätzungsweise mit rd. 9 Mio. Verletzten jährlich zu rechnen; im Jahr 1995 sind 39600 Personen an nichtnatürlichen Todesursachen gestorben (Tab. 1).

\section{Mortalität infolge von Verletzungen}

Der Anteil nichtnatürlicher Sterbefälle an allen Sterbefällen beträgt insgesamt zwar nur $5 \%$, im Kindes- und Jugendalter sowie im jüngeren Erwachsenenalter (etwa von zehn bis 25 Jahre) dominieren die nichtnatürlichen Todesursachen aber das gesamte Sterbegeschehen; bei den 15- bis 25jährigen Männern, vor allem wegen der großen Unfallhäufigkeiten, in etwa dreiviertel der Sterbefälle (Abb. 1). In der Rangfolge der Todesursachenklassen belegen sie hinter den Krankheiten des Kreislaufsystems, den bösartigen Neubildungen, den Krankheiten der Atmungs- und Verdauungsorgane den 5. Rangplatz. Beim vorzeitigen Verlust an potentiellen Lebensjahren (Altersbereich ein bis 65 Jahre) liegt die nichtnatürliche Sterblichkeit insgesamt weit vor den bösartigen Neubildungen und den Herz-Kreislaufkrankheiten. Allein die Todesursachengruppe Unfälle verzeichnet ebenso hohe Verluste wie die gesamte Klasse der HerzKreislaufkrankheiten. Der Grund dafür ist im sehr viel geringeren mittleren Sterbealter bei den nichtnatürlichen Todesursachen zu suchen. Bei Mord und KfzUnfall ist das Sterbealter am niedrigsten (Tab. 2).

Im Zeitverlauf (Abb. 2) ist in West und Ost sowie bei beiden Geschlechtern ein rückläufiger Trend der nichtnatürlichen Sterblichkeit zu erkennen, wobei die Sterblichkeitswerte in den neuen Bunreich ein bis $65 \mathrm{Jahre}$ desländern noch beträchtlich über denen der alten Bundesländer liegen, jedoch eine größere Regression erkennen lassen. In den neuen Bundesländern war zwischenzeitlich von 1989 zu 1990/91 ein deutlicher Anstieg der Unfallsterblichkeit zu verzeichen. Der anschließende Rückgang führte bei beiden Geschlechtern zu niedrigeren Werten als vorher. Bemerkenswert ist der Anstieg der Sterblichkeit an Mord und Totschlag sowie unbekannter Ursache in den neuen Ländern. Demgegenüber ist die Suizidsterblichkeit rückläufig.

Der tendenzielle Rückgang der Sterblichkeit an nichtnatürlichen Todesursachen ist nicht zwangsläufig auch ein Indikator für eine sich verringernde Verletztenhäufigkeit sondern scheint eher Folge besserer medizinischer Versorgung sowie geringerer Verletzungsschwere zu sein. Die steigende Häufigkeit an Mord und Totschlag ist nicht nur im Osten ein Indiz für zunehmende Gewaltanwendung im gesellschaftlichen Miteinander. In dem Sachstandsbericht 1993/94 »Gesundheit für alle« des europäischen Regionalbüros der WHO wird festgestellt, daß mit dem zunehmenden wirtschaftlichen Druck und den wachsenden sozialen Spannungen in der gesamten Region die persönliche Sicherheit immer stärker gefährdet er-

Tabelle 2: Nichtnatürliche Sterbefälle 1995 im Vergleich mit den häufigsten Todesursachen (Gestorbene, Mortalitätsrate, PYLL*, mittleres Sterbealter)

\begin{tabular}{|c|c|c|c|c|c|}
\hline & ICD-Nummer & absolut & $\begin{array}{r}\text { Gestorbene } \\
\text { je } 100000 \text { der } \\
\text { Bevölkerung }\end{array}$ & $\begin{array}{r}\text { PYLL } \\
\text { je } 100000 \text { der } \\
\text { Bevölkerung }\end{array}$ & $\begin{array}{r}\text { mitcleres } \\
\text { Sterbealter } \\
\text { (Jahre) }\end{array}$ \\
\hline $\begin{array}{l}\text { Verletzungen/ } \\
\text { Vergiftungen } \\
\text { (nichtnatürl. } \\
\text { Todesursachen) } \\
\text { darunter }\end{array}$ & Klasse XVII & 39600 & 48,2 & 4500 & \\
\hline $\begin{array}{l}\text { Unfall } \\
\text { dar. Kfz-Unf. }\end{array}$ & $\begin{array}{l}\text { E800-E949 } \\
\text { E810-E825 }\end{array}$ & $\begin{array}{r}23800 \\
6800\end{array}$ & $\begin{array}{l}29,2 \\
11,9\end{array}$ & $\begin{array}{l}2800 \\
1900\end{array}$ & $\begin{array}{l}50 \\
41\end{array}$ \\
\hline Suizid & E950-E959 & 12900 & 15,6 & 1200 & 56 \\
\hline Mord & E960-E969 & 1200 & 1,3 & 200 & 40 \\
\hline $\begin{array}{l}\text { Krankheiten } \\
\text { des } \\
\text { Kreislaufsystems }\end{array}$ & Klasse VII & 429400 & 525,8 & 2900 & 80 \\
\hline $\begin{array}{l}\text { Bösartige } \\
\text { Neubildungen }\end{array}$ & Klasse II & 197700 & 242,2 & 3900 & 72 \\
\hline $\begin{array}{l}\text { Krankheiten der } \\
\text { Atmungsorgane }\end{array}$ & Klasse VIII & 53800 & 65,6 & 400 & 80 \\
\hline $\begin{array}{l}\text { Krankheiten der } \\
\text { Verdauungsorgane }\end{array}$ & Klasse IX & 41800 & 51,2 & 2000 & 70 \\
\hline
\end{tabular}

\#) PYLL: potential years of life lost: vorzeitiger Verlust an potentiellen Lebensjahren, hier im Altersbe- 


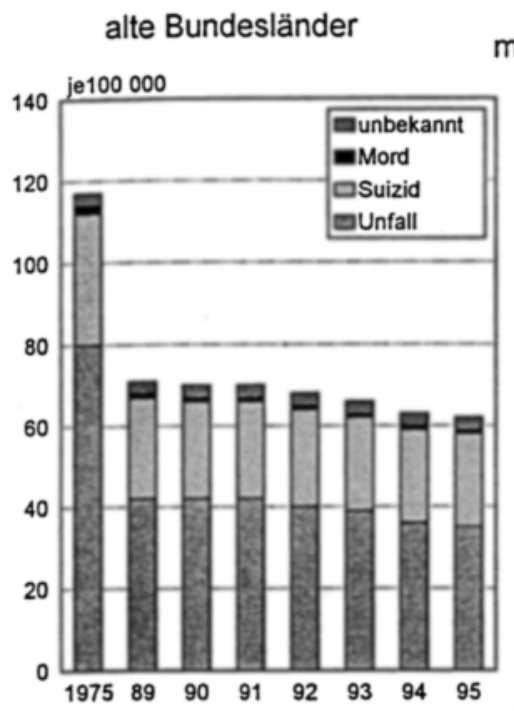

männlich
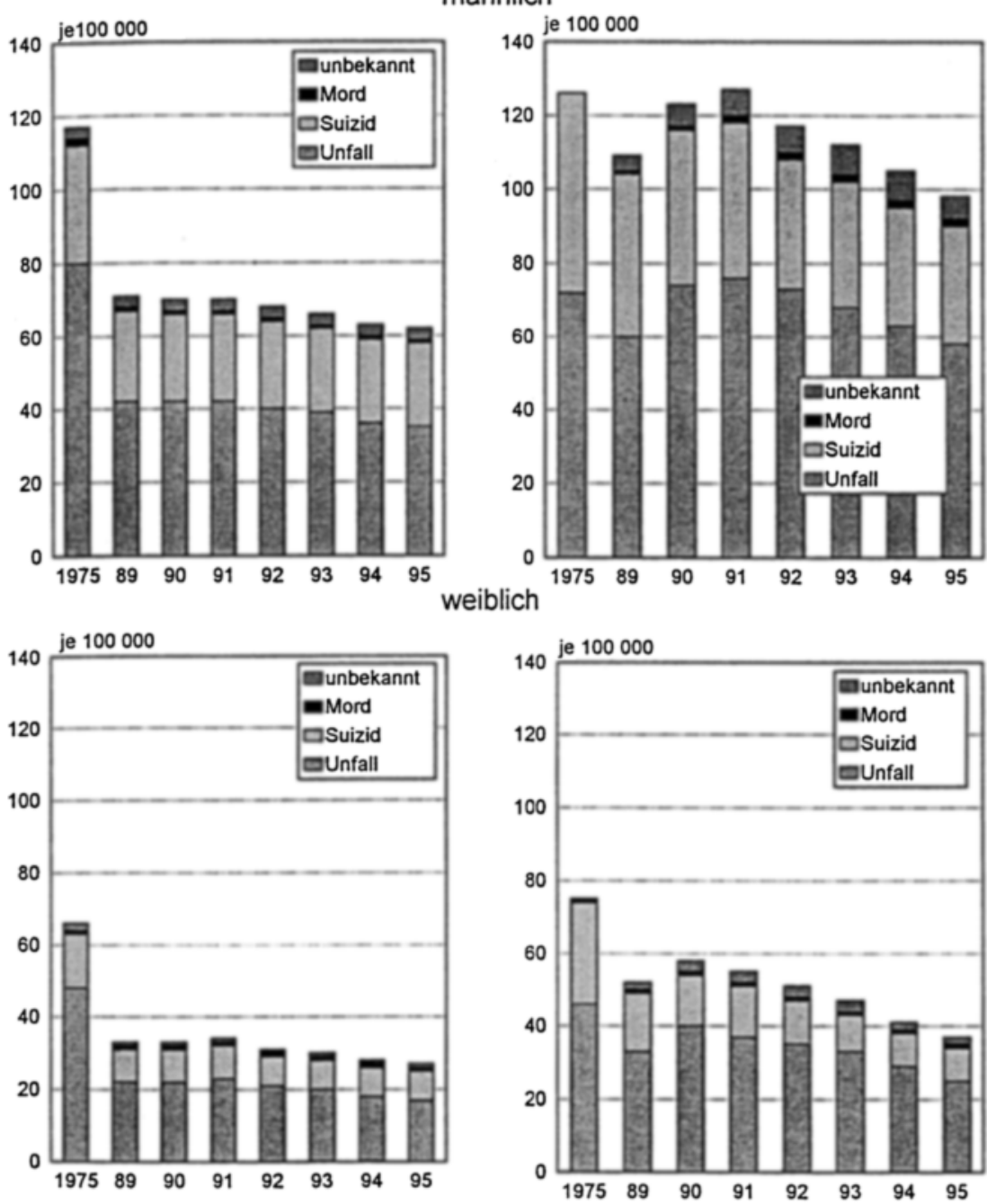

Standardisiert auf die deutsche Bevölkerung 1989

Abbildung 2: Nichtnatürliche Sterblichkeit 1975-1995 - Gestorbene je 100000 der Bevölkerung.

scheint. In fast allen Ländern ist die Lebensbedrohung größer als je zuvor, auch in der Bundesrepublik Deutschland sind die Tötungsdelikte häufiger geworden.

\section{Nichtnatürliche Sterblichkeit nach Ursachen, Alter, Geschlecht und Verletzungsart}

Rund $61 \%$ aller nichtnatürlichen Sterbefälle werden durch Unfälle verursacht, $33 \%$ durch Suizide, $2 \%$ durch Mord und Totschlag. Bei $4 \%$ der nichtnatürlichen Todesfälle ist unklar, welcher der drei Ursachengruppen sie zuzuordnen sind (Abb. 3). Schlüsselt man die Unfälle nach dem Ort des Entstehens auf, so wird deutlich, daß das Unfallgeschehen entgegen landläufigen Vorstellungen nicht von Verkehrs- und
Arbeitsunfällen, sondern von Hausund Freizeitunfällen bestimmt wird. Das zeigt sich sowohl bei den Gestorbenen als auch bei den Verletzten. In den
Altersgruppen von zehn bis 55 Jahre dominieren bei den männlichen Personen die Verkehrsunfälle.

An einer nichtnatürlichen Todesursache sterben wesentlich öfter männliche als weibliche Personen, in einigen Altersgruppen doppelt so häufig; das trifft ebenso auf die cinzelnen Ursachengruppen zu. Bei den männlichen Personen ist auch der Anstieg ab 15 Jahre deutlich größer als bei den weiblichen Personen, in den folgenden Altersgruppen ist ein annähernd gleicher Verlauf auf unterschiedlichem Niveau zu erkennen (Abb. 4). Unfälle sind zwar bei beiden Geschlechtern die häufigste Ursache von tödlich ausgehenden Verletzungen, im Alter von etwa 30 bis zu 70 Jahren erreichen aber die Suizide ähnliche Häufigkeiten. Mord, in diesem Falle Kindesmißhandlungen mit tödlichem Ausgang, weist bei beiden Geschlechtern im Säuglingsalter die höchsten Werte auf.

Die häufigsten zum Tode führenden Verletzungen sind sowohl bei den männlichen als auch bei den weiblichen Personen die Frakturen (Tab. 3). Fast die Hälfte der Frakturen männlicher Personen sind Schädelfrakturen. Schädelhirntraumen (Schädelfrakturen und intrakranielle Verletzungen zusammen, ICD-Nr. 800-804 und ICD-Nr. 850854) machen rund $40 \%$ der tödlichen Verletzungen der Männer aus, insbesondere sind die jungen Männer im Alter von etwa 15 bis 35 Jahre betroffen. Rund $60 \%$ der Frakturen weiblicher Personen sind Oberschenkelhalsfrakturen. Sie stehen ab 75 Jahre an der Spitze der weiblichen Sterbefälle infolge von Verletzungen und sind die häufigste Einzeltodesursache der nichtnatürlichen Mortalität bei den Frauen. Die häufigste Einzeltodesursache bei den Männern ist die traumatische Asphyxie (TU-Nr. 994.7), vor allem in den Altersgruppen $a b 20$

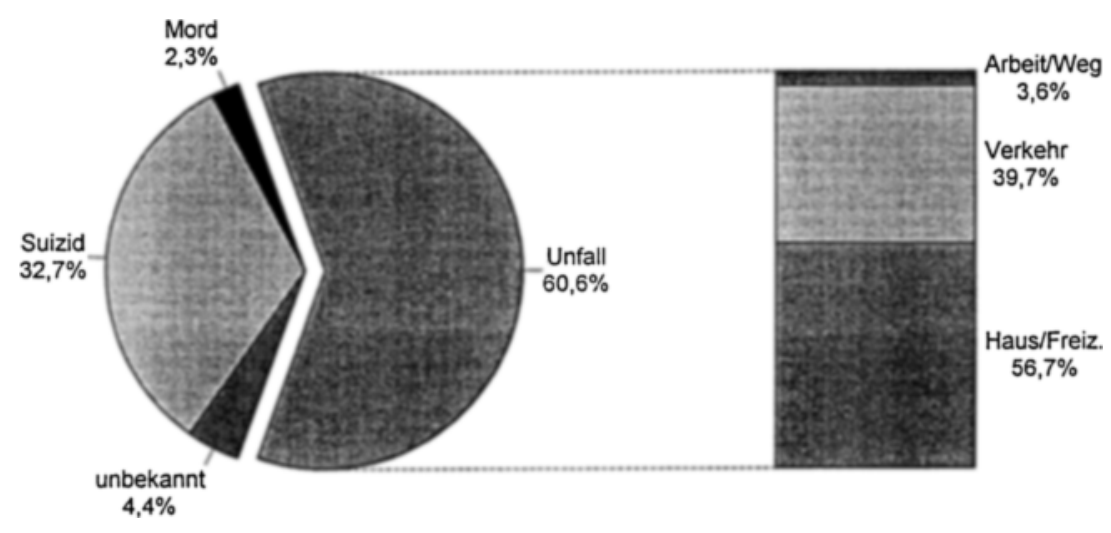

Abbildung 3: Nichtnatürliche Sterbefälle 1995 nach den Ursachen. 

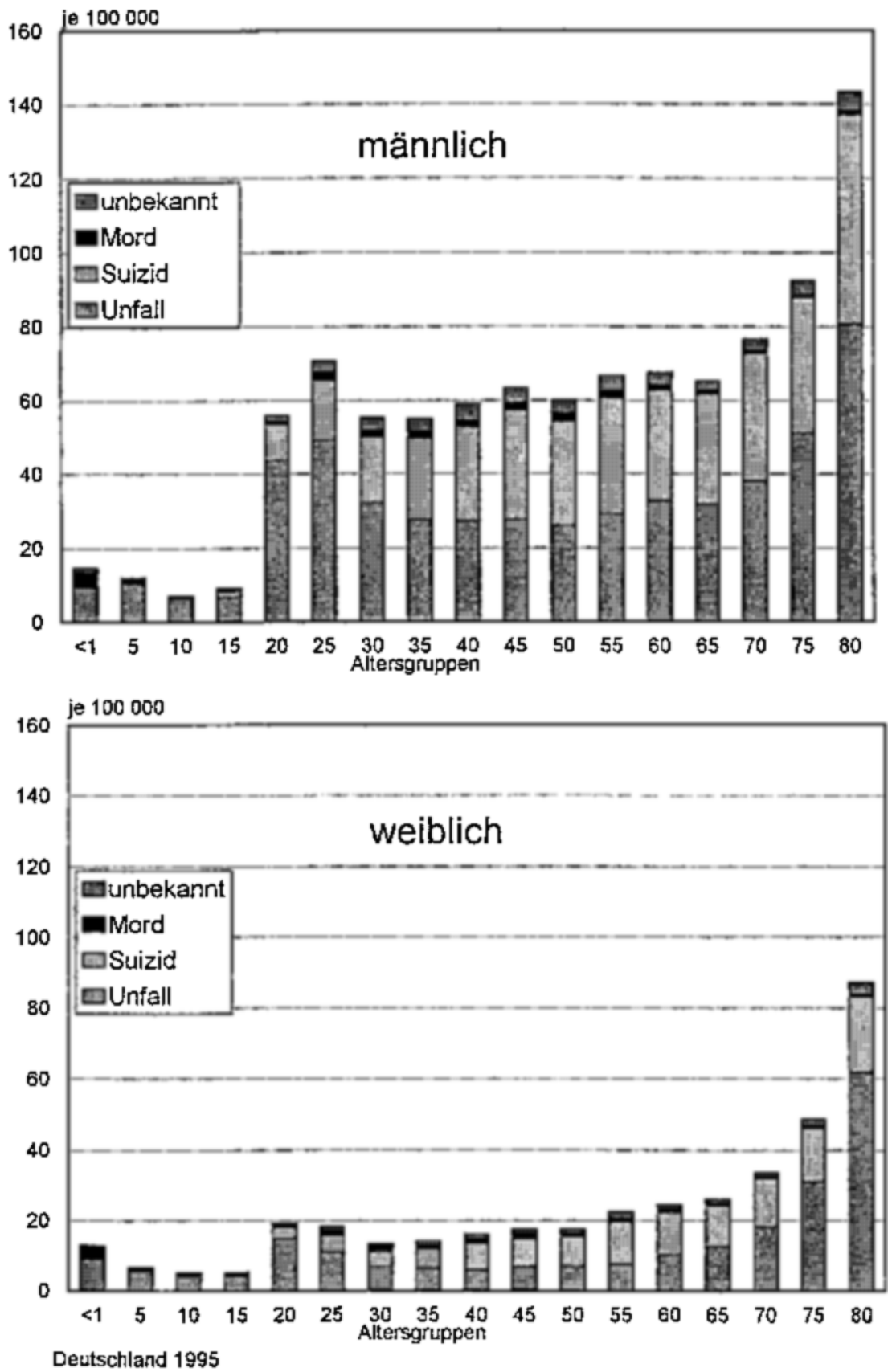

Abbildung 4: Nichtnatürliche Sterbefälle 1995 je 100000 der Bevölkerung nach Ursachen und Altersgruppen.

Jahre. Es ist zu vermuten, daß die Ursache in Suiziden mit der sogenannten harten Methode »Erhängen« (E 953.0) zu suchen ist.

\section{Morbidität infolge von Verlet- zungen}

Für die Darstellung der Morbidität stehen Daten aus der Krankenhausdiagnosenstatistik zur Verfügung. Die Dokumentation erfolgt nach den Diagnosen figkeit und die Struktur der Verletzungen fehlen, die gleichzeitig spezifische Gefährdungen sowie Ansatzpunkte für die Prävention aufzeigen können. Das betrifft vorrangig die Unfälle. Aus Vergleichen der Unfallsterbeziffern mit Verletztenziffern aus Studien (Institut für Medizinische Statistik und Datenverarbeitung, Bundesanstalt für $\mathrm{Ar}$ beitsschutz) geht $\mathrm{z}$. B. hervor, daß zwischen Verletzten- und Gestorbenenhäufigkeiten bestimmter Unfallursachen große strukturelle Unterschiede bestehen. So sind Stürze, die im Sterbegeschehen erst im höheren Alter von Bedeutung sind, die häufigste Verletzungsursache im Kindesalter. Detailliertes Wissen um die Häufigkeit und die Ursachenstruktur auch von nichttödlichen Verletzungen ist demzufolge für eine gezielte Unfallprävention unverzichtbar. Dafür wäre eine periodische Erfassung, Auswertung und Analyse von entsprechenden repräsentativen Daten nötig, auch um die Wirkung von Präventionsmaßnahmen zu evaluieren. Aus den künftigen nationalen Gesundheitssurveys sind wichtige diesbezügliche Informationen zu erwarten.

\section{Häufigkeit von Krankenhausbehandlungen}

Im Jahre 1994 waren 1,56 Mio. stationäre Behandlungen wegen Verletzungen und Vergiftungen $\mathrm{zu}$ verzeichnen, 840000 bei den männlichen und 720000 bei den weiblichen Personen. Das bedeutet, daß etwa jede fünfte Person der 9 Mio. Verletzten längere Zeit in einem Krankenhaus behandelt werden mußte. Der Anteil an allen stationären Behandlungen beträgt bei den Männern $13 \%$ und bei den Frauen $9 \%$. Verletzungen und Vergiftungen sind etwa gleich häufig wie Neubildungen die Ursache einer Krankenhausbehandlung. Sie belegen mit diesen gemeinsam den zweiten Rangplatz hinter den Krankheiten des Kreislaufsystems. Aus Verletzungen resultierten 19 Mio. Krankenhausbehandlungstage, das sind $11 \%$ aller Behandlungstage. Im Jahre 1994 waren wegen Verletzungen oder Vergiftungen täglich rd. 5100 Krankenhausbetten belegt.

\section{Krankenhausbehandlungen nach Alter, Geschlecht und Verletzungsart}

Sowohl die altersspezifischen Behandlungshäufigkeiten als auch die Anteile der Krankenhausfälle infolge von Ver- 
Tabelle 3: Nichtnatürliche Sterbefälle nach ausgewählten Todesursachen der Klasse XVII

\begin{tabular}{|c|c|c|c|c|c|}
\hline \multirow[t]{2}{*}{ Todesursache } & \multirow{2}{*}{$\begin{array}{l}\text { Nr. der } \\
\text { ICD-9 }\end{array}$} & \multicolumn{4}{|c|}{ Anteil in Prozent } \\
\hline & & v. K1. XVII & v. $800-829$ & v. Kl. XVII & v. $800-829$ \\
\hline Sterbefälle insgesamt & $800-999$ & $100 \%$ & & $100 \%$ & \\
\hline dar. Frakturen & $800-829$ & $26 \%$ & & $46 \%$ & \\
\hline dar. Schädelfraktur & $800-804$ & $12 \%$ & $47 \%$ & $7 \%$ & $16 \%$ \\
\hline $\begin{array}{l}\text { Oberschenkel- } \\
\text { halsfraktur }\end{array}$ & 820 & $5 \%$ & $20 \%$ & $27 \%$ & $59 \%$ \\
\hline $\begin{array}{l}\text { intrakranielle } \\
\text { Verletzungen }\end{array}$ & $850-854$ & $16 \%$ & & $12 \%$ & \\
\hline $\begin{array}{l}\text { traumatische } \\
\text { Asphyxie und } \\
\text { Strangulation }\end{array}$ & 994.7 & $21 \%$ & & $10 \%$ & \\
\hline
\end{tabular}

falltagen, woraus kostenmäßig etwa 7 Mrd. DM resultieren. Zuzüglich Kosten für Rehabilitation ergeben sich für die medizinische Betreuung einschließlich Arbeitsunfähigkeit Kosten in Höhe von schätzungsweise $22 \mathrm{Mrd}$. DM pro Jahr. Detaillierte Kostenschätzungen für die Hauptursachengruppen von Verletzungen, Unfall, Suizid, Gewalteinwirkungen durch eine andere Person lassen sich wegen fehlender Dokumentation nicht vornehmen.

\section{Resümee}

Verletzungen sind wegen ihrer Häufigkeit und der Besonderheiten ihrer Ent- letzungen und Vergiftungen an allen Krankenhausbehandiungsfällen bieten ein ähnliches Bild wie bei den Gestorbenen (Abb. 5,6). Die höchsten Werte verzeichnen die männlichen Personen der Altersgruppen 15 bis 25 Jahre und die weiblichen Personen ab 75 Jahre. Männliche Personen weisen bis etwa 65 Jahre zum Teil beträchtlich höhere Werte auf als weibliche Personen. In den höheren Altersgruppen dominieren vor allem bei den Behandlungshäufigkeiten die Frauen. Es ist zu vermuten, daß die Verletzungen bei den jüngeren Männern, die stationär behandelt werden müssen, nicht nur die Folge von Unfällen, sondern auch von anderweitigen Gewalteinwirkungen sind, während sie bei den Frauen im höheren Alter von Unfällen verursacht werden.

Die häufigsten Verletzungen, die eine stationäre Behandlung erforderlich machen, sind bei beiden Geschlechtern, wie bei den Gestorbenen, die Frakturen, wobei die Frakturen der unteren Extremitäten, zu denen die Oberschenkelhalsfrakturen gehören, an der Spitze stehen (Tab. 4). Die häufigste Einzelursache ist bei den männlichen Personen die Gehirnerschütterung (Diagnose Nr. 850) und bei den Frauen die Oberschenkelhalsfraktur (Diagnose Nr. 820).

\section{Kosten}

Die jährlichen Kosten für die stationäre Behandlung von Verletzungen betragen etwa 9 Mrd. DM. Nach Expertenschätzungen machen die Kosten für die nichtstationäre medizinische Behandlung von Verletzten etwa den gleichen Betrag aus wie die Kosten für Krankenhausbehandlungen. Verletzungen verursachen darüber hinaus jährlich 3 bis $4 \mathrm{Mio}$. Arbeitsunfähigkeitsfälle mit 65 Mio. Aus-

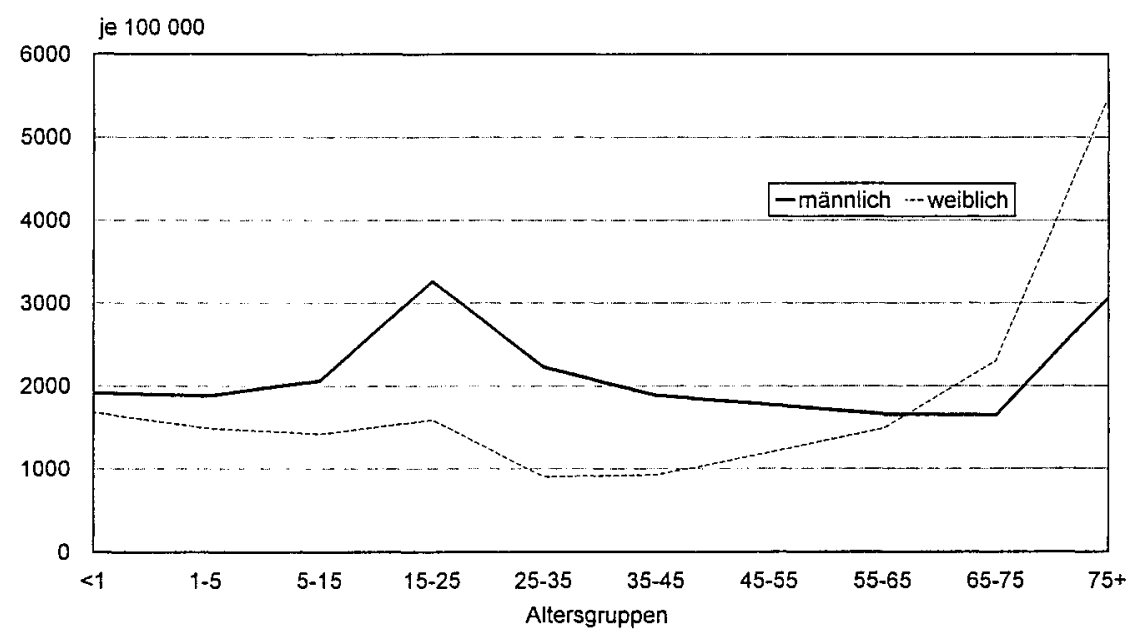

Abbildung 5: Verletzungen und Vergiftungen - Krankenhausbehandlungsfälle $1994 \mathrm{je}$ 100000 der Bevölkerung.

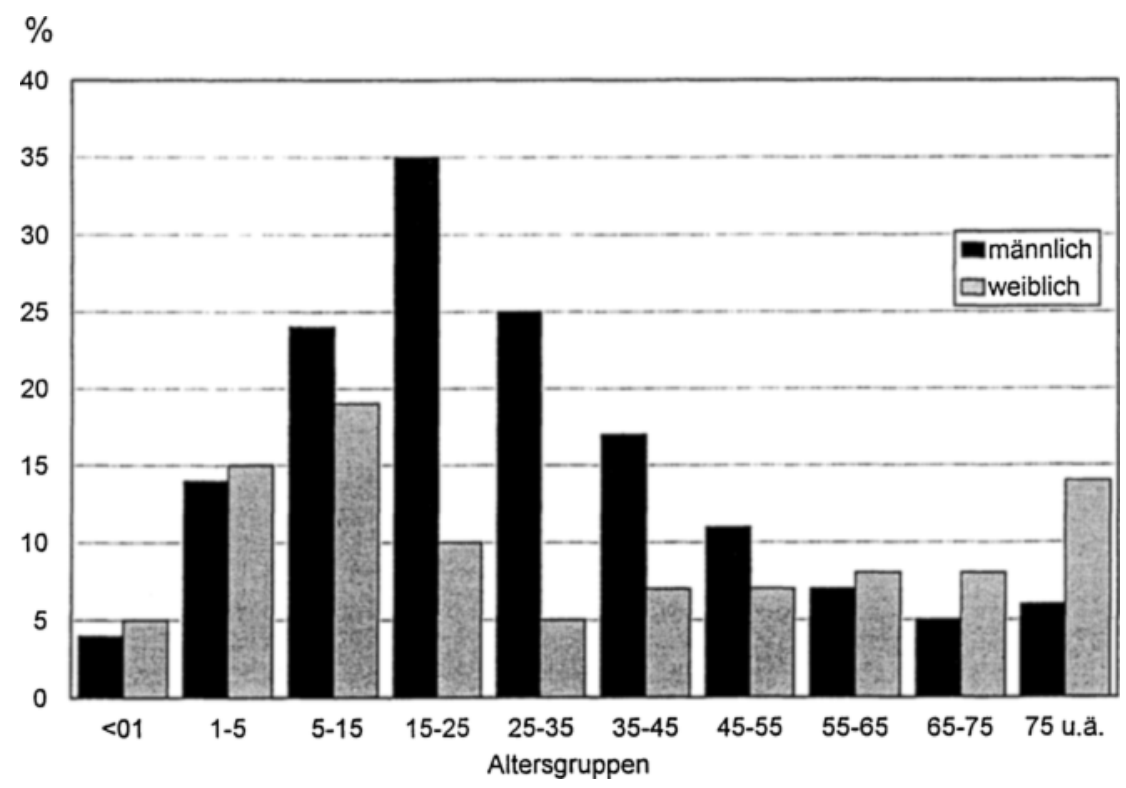

Abbildung 6: Anteile der Verletzungen und Vergiftungen an den Krankenhausfällen der Altersgruppen. 
Tabelle 4: Häufigkeit von stationär behandelten Frakturen nach dem Geschlecht (Krankenhausfälle je 100000 der Bevölkerung)

\begin{tabular}{lccc}
\hline Art der Fraktur & Nr. ICD-9) & $\mathrm{m}$ & Häufigkeiten \\
\hline Fraktur der unteren Extremität & $820-829$ & 384,4 & 475,5 \\
Fraktur der oberen Extremität & $810-819$ & 238,8 & 238,8 \\
Fraktur von Hals und Rumpf & $805-809$ & 124,3 & 122,9 \\
Klasse XVII & $800-999$ & 2120,5 & 1718,0 \\
\hline
\end{tabular}

(Quelle: RKI - GBE)

stehung ein Gesundheitsproblem insbesondere im Kindes- und jüngeren $\mathrm{Er}$ wachsenenalter. In diesem Alter dominieren sie das Sterbegeschehen und haben vor allem bei den männlichen Personen einen bedeutenden Anteil auch am Morbiditätsgeschehen. Im öffentlichen Bewußtsein ist jedoch nur ein geringer Teil, die Verkehrsunfälle und die Gewalttätigkeiten, präsent, meist ohne Bezug auf Prävention. Aber Verletzungen lassen sich in gewissem Umfang verhüten. Vor allem sind die Verletzungen durch Unfälle einer Prävention zugänglich. Die meisten Unfälle gelten als vermeidbar, und für Arbeits- und Verkehrsunfälle existieren entsprechende, Wirkung zeigende Präventionskonzepte. Der Verhütung von Haus- und Freizeitunfällen, dazu gehören auch die Unfälle der Kinder und der älteren Personen, wird hierzulande bisher wenig Auf- merksamkeit zuteil. Für die Senkung der Sterbe- und Verletzungshäufigkeiten ist aber insbesondere die Prävention von Haus- und Freizeitunfällen wichtig, gegründet auf entsprechende Informationen. Beispielgebend in der Unfallprävention sind u. a. Schweden, Norwegen, Österreich, die gesellschaftlich und gesundheitspolitisch unterstützte und geförderte Prävention von großer Wirksamkeit betreiben. Auch die WHO unterstützt solche Projekte, wie »Safe Community-Network«, »Helmet-Initiative

Die häufiger werdenden Gewalttätigkeiten gegen andere Personen sind Ausdruck wachsender sozialer Spannungen und sozialer Ausgrenzung vor allem der jungen Generation. Ihre Prävention ist eine gesellschaftliche Aufgabe von hohem Rang.
Literatur:

- Bergmann, K. E., Baier, W., Casper, W., und Wiesner, G. (Hrsg.): Entwicklung der Mortalität in Deutschland von 1955-1989. BGA-Schriften $5 / 92$.

- Casper, W., Wiesner, G., und Bergmann, K.E. (Hrsg.): Mortalität und Todesursachen in Deutschland. RKI-Heft 10, 1995.

- Casper, W.: Die Unfallmortalität in der Bundesrepublik Deutschland. Bundesgesundheitsbl. 36 (1993) 281-284

- Casper, W.: Unfälle. In: Die Gesundheit der Deutschen. Ein Ost-West-Vergleich. Berlin: Institut für Sozialmedizin und Epidemiologie des Bundesgesundheitsamtes, SozEp Hefte 4/1994.

- Casper, W.: Verletzungen und Vergiftungen. In: Das Gesundheitswesen, Jahresgesundheitsbericht 1989 für das Gebiet der ehemaligen DDR. Bd. 25, 1990.

- Miller, T. E., and Lestina, D. C.: Patterns in US Medical Expenditures and Utilization for Injury 1987. American Journal of Public Health. Vol. 86, No. 1.

- Statistisches Bundesamt: Mikrozensus Gesundheitszustand der Bevölkerung.

- Statistisches Bundesamt: Gesundheitsbericht, Kapitel »Verletzungen - Überblick «.

- WHO: Gesundheit in Europa. „Gesundheit für allex: Sachstandsbericht 1993/94. Europäische Schriftenreihe Nr. 56. Regionale Veröffentlichungen der WHO. Kopenhagen.

- Bundesanstalt für Arbeitsschutz: Unfallgeschehen in Heim und Freizeit. Schriftenreihe, Sonderschrift S30 (1990) und S33 (1993).

Anschrift der Verfasserin

Dr. W. Casper, Robert Koch-Institut, Fachbereich Nicht übertragbare Krankheiten und Gesundheitsberichterstattung, General-Pape-Straße 62-64, 12101 Berlin 